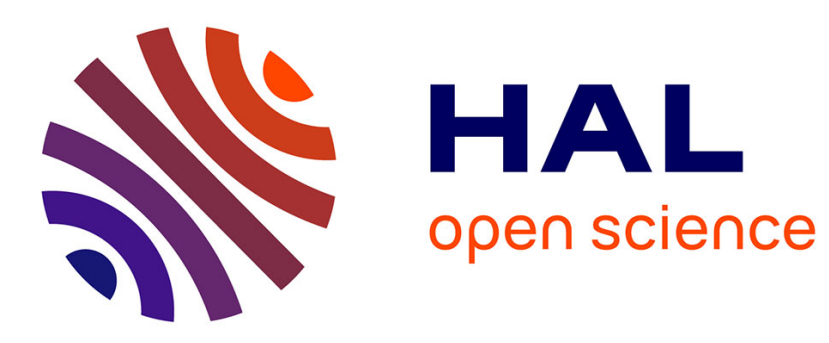

\title{
Parabolic manifolds in the scattering map and direct quantum processes
}

\author{
B. Dietz, M. Lombardi, T. H. Seligman
}

\section{To cite this version:}

B. Dietz, M. Lombardi, T. H. Seligman. Parabolic manifolds in the scattering map and direct quantum processes. Journal of Physics A: Mathematical and Theoretical, 1996, 29, pp.L95-L99. 10.1088/03054470/29/5/003 . hal-00972665

\section{HAL Id: hal-00972665 \\ https://hal.science/hal-00972665}

Submitted on 3 Apr 2014

HAL is a multi-disciplinary open access archive for the deposit and dissemination of scientific research documents, whether they are published or not. The documents may come from teaching and research institutions in France or abroad, or from public or private research centers.
L'archive ouverte pluridisciplinaire HAL, est destinée au dépôt et à la diffusion de documents scientifiques de niveau recherche, publiés ou non, émanant des établissements d'enseignement et de recherche français ou étrangers, des laboratoires publics ou privés. 


\title{
LETTER TO THE EDITOR
}

\section{Parabolic manifolds in the scattering map and direct quantum processes}

\author{
B Dietz $\dagger$, M Lombardi $\ddagger$ and T H Seligman $\dagger$ \\ $\dagger$ Laboratorio de Cuernavaca, Instituto de Física, UNAM, Cuernavaca, Mexico \\ $\ddagger$ Laboratoire de Spectrométrie Physique, Université de Grenoble, Grenoble, France
}

Received 9 November 1995

\begin{abstract}
We analyse the quantum effects of parabolic manifolds in Jung's iterated scattering map. For this purpose we consider the classical map proposed previously to be the exact classical analogue of Rydberg molecules calculated with the approximations relevant to the multichannel quantum defect theory for energies above the ionization threshold. The part corresponding to positive electron energies can be viewed as a Jung scattering map without the trivial direct processes. This map contains a parabolic manifold of fixed points which gives rise to a regular series of quantum states which behave very much like eigenchannels that miss the target.
\end{abstract}

The classical behaviour of the scattering map introduced by Jung [1] is directly responsible for the statistical properties of the $S$-matrix at fixed energy [2,3]. As the chaoticity of the Jung map is necessary but not sufficient for topological chaos in the scattering process, one can reasonably ask how relevant the chaoticity of the Jung map and the corresponding random matrix behaviour of the $S$-matrix actually are. An important problem in this context is the elimination of trivial direct processes associated with particles that miss the target $[4,3]$.

The purpose of this letter is to exemplify how the classical structure of the scattering map has immediate and drastic consequences for the classification of direct processes. We shall see that in the chaotic region of the map parabolic manifolds of fixed points lead to a situation where one channel becomes direct, and in some sense trivial, despite the fact that it is nicely embedded in the chaotic region of the Jung map. The example we use is electron scattering off a molecule ion (core) near threshold in a situation that physically corresponds to spontaneous ionization of a Rydberg molecule; the approximations typical for calculations in such systems are used.

The Rydberg molecule was analysed in previous papers [5-8] using multichannel quantum defect theory (MQDT) [9]. Lombardi et al proposed a classical map, the quantization of which is exactly solved by the MQDT [6]. This close analogy allows interesting studies on the behaviour of quantum signatures of classical chaos [8, 10]. As the map contains approximations with respect to the true Rydberg molecule, it may not be realistic in all cases. Indeed, it is practically opposite to the approximations in recent calculations [11]. On the other hand experimental verification exists for small electron angular momenta and is not, in principle, excluded for systems with large organic cores and multilaser excitation. Furthermore, the approximate system deserves being studied for its own sake precisely because we can compute the corresponding quantum results without approximation. With 
this proviso we shall in this letter refer to the approximate classical system as the Rydberg molecule.

In [6] the Rydberg molecule is described classically as a map of the unit sphere onto itself. Points on this sphere correspond to the orientations of the electron orbital angular momentum whose absolute value is approximated to be constant. This sphere constitutes a Poincare surface of section for the entire problem, the dynamics of which is described completely by this section.

Due to conservation of total angular momentum, the angular momentum of the core must change, and consequently the energy of the core will change. Energy conservation in turn obliges the radial function of the electron to change. If the total energy is just slightly above the ionization threshold for the lowest core angular momenta allowed, we will find points on this sphere for which the electron energy is positive and others for which it is negative. The zero electron energy points form a circle that separates the two sets. Therefore, we can describe a scattering process by initiating the map with a point in the positive electron energy region and terminating it whenever we hit this region again. Ionization processes would correspond to starting at some point of negative electron energy and stopping when the positive energy region is hit for the first time.

The map on the sphere is defined in the molecular reference frame $[6,8]$, where the $z$-axis is chosen along the molecular axis and the $x$-axis along the angular momentum of the molecule ion. In this reference system the direction of the electron's angular momentum is given in terms of the angles $\theta$ and $\phi$ to the $z$-axis and the $x$-axis, respectively. For negative electron energies, that is, while the electron is trapped temporarily or forever by the core on a very eccentric orbit, each iteration of the Poincaré map consists of two consecutive rotations:

(i) In the collision region the electron's angular momentum experiences a rotation about the molecular $z$-axis due to the cylindrical symmetry of the core. Further symmetry arguments require that this rotation be odd in $\theta \rightarrow \pi-\theta$. Then the simplest choice for the rotation is $\delta \phi=K \cos \theta$, where $K$ denotes the coupling strength between the electron and the molecule ion. Since the time the electron spends in the collision region is negligible compared to the time it spends in the asymptotic region, we may insert for $\theta$ the angle corresponding to the angular momentum direction of the electron when it enters the collision region, i.e. we may assume that the interaction effect is a kick by $\delta \phi$. Conservation of angular momentum and energy entails an additional $\delta \phi$ (but no change of $\theta$ ) and the above-mentioned exchange of energy between the core and the electron [6].

(ii) In the asymptotic region far away from the core the electron's orbit is Coulombic and the energy and angular momenta of the electron and the core are conserved. Yet in the rotating molecular frame the electron's angular momentum experiences a rotation about the $x$-axis.

The positive electron energy region is a cap about the positive $x$-axis in the reference system defined above. On this cap we may define a Jung scattering map, by simply using the same angular momentum orientation, i.e. the exit point on the cap, as a new entry point. This seems rather unfamiliar, but due to the fact that the section is given in terms of angular momenta, it can be shown to be the appropriate definition of the Jung map for this system [1]. Note that if the electron is not trapped by the molecule ion, its angular momentum only experiences a rotation about the $z$-axis by an angle $\delta \phi=K \cos \theta$. This definition has the very attractive property that the corresponding manifold is compact, and consequently the $S$-matrix will have finite dimension. This saves us all the problems with truncation of an infinite-dimensional $S$-matrix [4].

Considering the simple dynamics of the Jung map we see immediately that a line of 
constant angle $\theta=\pi / 2$ is mapped onto itself, because the shift $\phi_{\text {new }}-\phi_{\text {old }}=K \cos \theta$ is zero, while the shift in $\theta$ is zero for the positive electron energy cap by the definition of the Jung map. We thus have a parabolic manifold of fixed points bisecting the positive energy cap at $\theta=\pi / 2$. Indeed, we can see in figures $1(a),(b)$ the fixed points of the Jung map consisting exclusively of the parabolic manifold. Note that the major part of the backside of sphere 1 $(b)$ does not belong to the Jung map; the fixed points of this area are not shown. The fixed points were searched by methods discussed in [12]. Note that for $K>2 \pi$ several parabolic manifolds may appear.
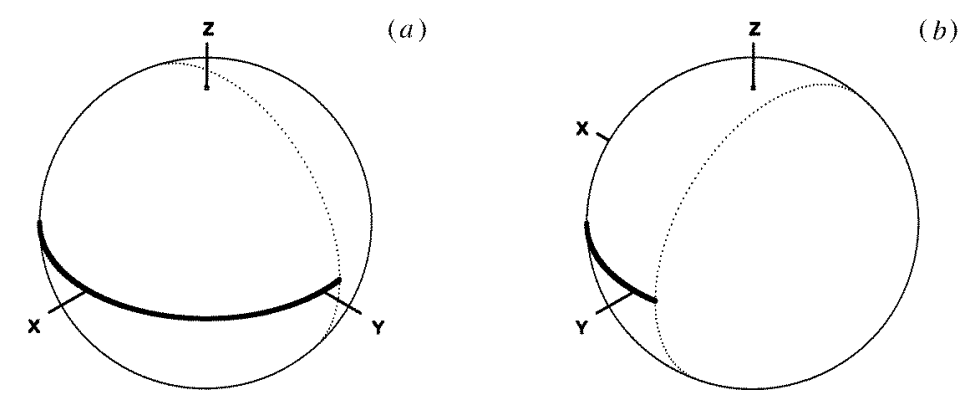

Figure 1. Fixed points for one iteration of the Jung map. The dotted curve is the limit of ionization.

Such orbits are known to play a crucial role, e.g. in the stadium where they give rise to the so-called bouncing ball states, that in turn cause non-universal behaviour in spectral statistics [13]. We expect analogous eigenstates of the $S$-matrix.

Our quantum treatment of this problem is not a direct quantization of the Jung map. Rather we deal with a quantization of the entire Poincare surface of section using the MQDT techniques [9]. We will define states on the entire sphere, from which the full quantum states of the system may be obtained. In figures $2(a),(b)$ we see the Husimi distribution for a state that follows the parabolic manifold of figure 1 quite closely. Figure $2(b)$ shows the backside of the sphere where this state vanishes except for the tips of the parabolic manifolds that go around to the back. Thus, this state does not seem to couple to the complicated structures of topological chaos of the temporarily trapped orbits described by the backside of the spherical surface of section. For comparison we show in figures $2(c),(d)$ the Husimi distribution of a typical eigenstate of the $S$-matrix.

The state mimicking the parabolic manifold has other very characteristic features. If we consider the spectrum of $S$ as a function of energy or some external parameter like the coupling strength $K$, it is useful to unwrap the spectrum, i.e. to follow a drift of eigenphases outside the interval $[0,2 \pi]$. Typically, such a drift exists for the eigenphases of any $S$-matrix. The spurious eigenchannels, not affected by the target, do not participate in this drift. Such eigenchannels should have been eliminated by our procedure. Yet figure 3 , which shows such an unwrapping, discloses that one such eigenphase persists. The Husimi distribution of the corresponding eigenchannel is precisely the one shown in figure 2. Despite the elimination of trivially uninteresting channels from the $S$-matrix we are thus stuck with one such channel, which we have to eliminate if we wish to obtain the spectral statistics of the $S$-matrix $[14,15]$ in accordance with random matrix expectations.

Summarizing, we may say that we found parabolic manifolds in the Jung map to have a very direct significance for the scattering process, as they lead to trivial direct channels even though they are embedded in the chaotic part of the map. This result is of direct 

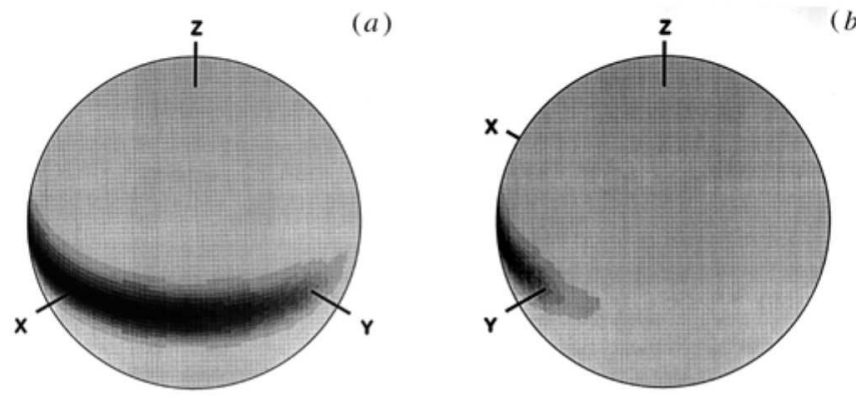

(b)
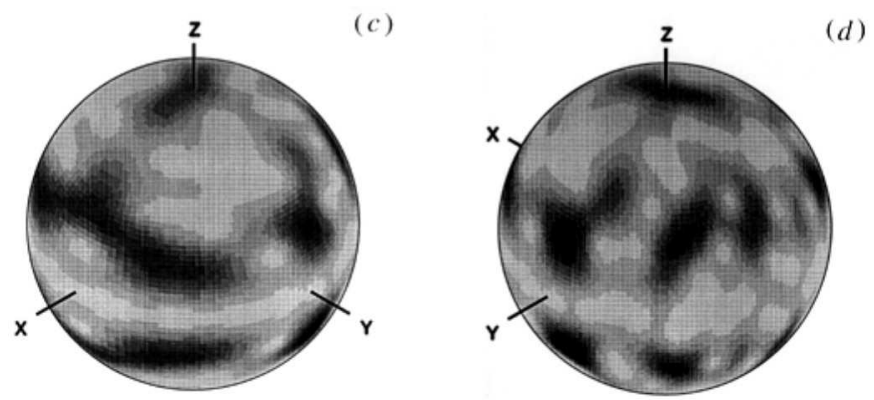

Figure 2. Husimi distributions of eigenstates. $(a)$ and $(b)$ show the peculiar quantum state scarred on the parabolic manifold discussed in the text; $(c)$ and $(d)$ show a typical quantum state.

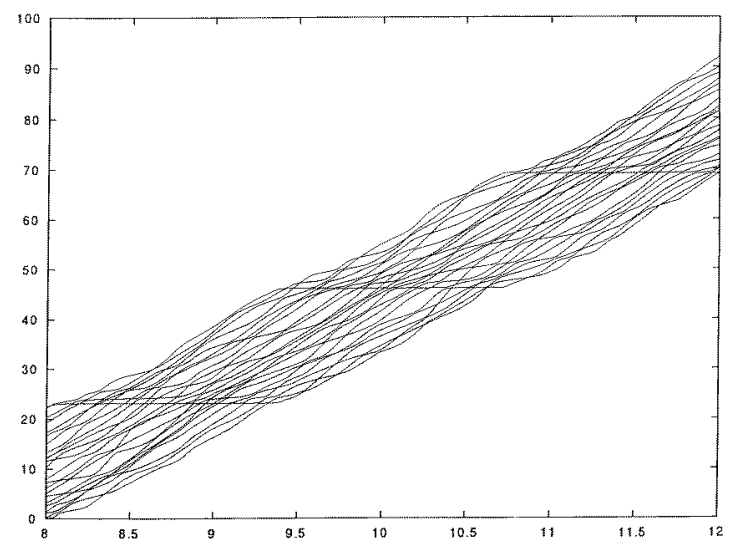

Figure 3. Eigenphases of the $S$-matrix as a function of energy (unwrapped). The nearly horizontal line which occurs three times corresponds to the parabolic manifold. Figure 2 gives the Husimi distribution of the eigenstate corresponding to the middle horizontal line.

importance if we wish to carry out an analysis along the lines of [3] where such channels have to be eliminated. Beyond that they show that semiclassical properties of the Jung map determine important parts of the quantum scattering process despite the fact that they do not contain the information about the presence or absence of topological chaos. 
One of the authors (BD) would like to thank the Alexander-von-Humboldt Stiftung for a scholarship. This work was supported by the University of Mexico DGAPA contract IN100491 and by the contract SC-101094 of CRAY Research and the University of Mexico.

\section{References}

[1] Jung C 1986 J. Phys. A: Math. Gen. 191345

[2] Seligman T H 1992 Proc. Wigner Symp. (Goslar 1991) (Singapore: World Scientific) Blümel R, Dietz B, Jung C and Smilansky U 1992 J. Phys. A: Math. Gen. 251483

Seligman T H 1995 Quantum Chaos ed G Casati and B Chirikov (Cambridge: Cambridge University Press) p 577

[3] Jung C and Seligman T H 1995 J. Phys. A: Math. Gen. 281507

[4] Smilansky U 1991 Chaos and Quantum Physics (Les Houches, Session LII) (Amsterdam: North-Holland)

[5] Bordas M C, Broyer M, Chevaleyre J, Labastie P and Martin S 1985 J. Physique 4627

Labastie P, Bordas M C, Tribollet B and Broyer M 1984 Phys. Rev. Lett. 521681

[6] Lombardi M, Labastie P, Bordas M C and Broyer M 1988 J. Chem. Phys. 893479

[7] Lombardi M, Pique J P, Labastie P, Broyer M and Seligman T H 1991 Comments At. Mol. Phys. 25345

[8] Lombardi M and Seligman T H 1993 Phys. Rev. A 473571

[9] Fano U 1970 Phys. Rev. A 2353

Seaton M J 1983 Rep. Prog. Phys. 46167

[10] Leyvraz F, Quezada J, Lombardi M and Seligman T H 1991 Phys. Rev. Lett. 672921

[11] Benvenuto F, Casati G and Shepelyansky D L 1994 Phys. Rev. Lett. 721818

[12] Lombardi M, Seligman T H and Leyvraz F to be published

[13] Gräf H D et al 1992 Phys. Rev. Lett. 691296

Shudo A and Shimizu Y 1990 Phys. Rev. A 426264

[14] Dietz B, Lombardi M and Seligman T H Phys. Lett. A submitted

[15] Dietz B, Lombardi M and Seligman T H to be published 